\title{
Analysis of the Optimal Battery Sizing for Plug-in Hybrid and Battery Electric Vehicles on the Power Consumption and V2G Availability
}

\author{
J. Van Roy, Student Member, IEEE, S. De Breucker, Student Member, IEEE, and J. Driesen, Member, IEEE
}

\begin{abstract}
The interest in electric vehicles (EVs) experiences a strong growth. Batteries of EVs will be charged at home, which means there will be an increase in the household power consumption. This impact on the distribution and transmission grid can be minimized by e.g. (i) a coordinated charging strategy and (ii) choosing an optimal battery size for each vehicle. A second drawback of EVs are the high cost and weight of the batteries. This paper proposes some allocation scenarios to allocate battery sizes to a fleet of plug-in hybrid (PHEV) and battery electric vehicles (BEVs). Based on statistical data of Flanders (northern region in Belgium), a fleet of vehicles with realistic driving patterns is created, which is used to choose the optimal battery size. Different allocation scenarios will be compared regarding the effect on the proportion electric driving, the extra household power consumption and the fleet availability for Vehicle-to-Grid (V2G) services.
\end{abstract}

Index Terms-Battery capacity, Electric vehicle, Plug-in hybrid electric vehicle, Utility factor, Vehicle-to-grid.

\section{INTRODUCTION}

$\mathbf{S}$ INCE a few years, the oil prices have been increasing strongly [1]. Together with the awareness of the climate problem, there is an increasing interest in electric vehicles (EVs). The cost and weight of batteries, which are used as storage for the electrical energy, are still a drawback for a high-scale commercialization of EVs [2]. These disadvantages call for an optimal battery sizing.

Hybrid electric vehicles (HEVs) combine an internal combustion engine (ICE) with an electric motor (EM). Today, mainly batteries are used to store the energy needed for the EM. The battery capacity for a HEV is very limited $(1-2 k W h)$. Therefore, the all-electric range (AER) is limited to a few kilometers. A HEV operates in charge sustaining mode (CS) to keep the state of charge ( $\mathrm{SoC}$ ) of the batteries at a nearly constant level.

Plug-in hybrid electric vehicles (PHEVs) are full HEVs, with the possibility to plug the vehicle into an electrical outlet to charge their batteries. PHEVs have an extended battery capacity to drive a limited purely electric distance [3]. They are characterized by PHEVx, with $x$ the total number of miles that can be driven electrically (e.g. a PHEV10 has an AER of 10 miles). During this distance, a PHEV operates in charge depletion mode (CD), in which the SoC of the battery is

J. Van Roy, S. De Breucker and J. Driesen are with the Department of Electrical Engineering (ESAT), Division ELECTA, Katholieke Universiteit Leuven, Kasteelpark Arenberg 10, bus 2445, 3001 Leuven-Heverlee, Belgium (e-mail: Juan.VanRoy@esat.kuleuven.be).

S. De Breucker is with VITO, Boeretang 200, 2400 Mol, Belgium. decreased. When the battery is depleted, a PHEV operates in CS-mode and becomes a HEV.

Battery electric vehicles (BEVs) only use an EM for propulsion. The energy resource (e.g. batteries) has to be well dimensioned, since it is important to have enough energy for all the vehicle trips between two charging periods. This paper discusses the battery sizing for PHEVs and BEVs.

\section{Problem Statement}

For PHEVs, many studies often work with a fixed battery capacity of around $10 \mathrm{kWh}$ (e.g. a useful capacity of $8.8 \mathrm{kWh}$ in [4]) or more. The charging of EVs will take place especially at home during the night. A battery with a useful capacity of e.g. $8.8 \mathrm{kWh}$ (and a charging efficiency of 90\% [5]) will approximately double the yearly average electricity consumption of a Flemish household $(3,500 k W h$ [6]) in case the battery has to be fully charged every night. In this case, a high penetration rate of PHEVs in a distribution grid will have a large impact on the electricity grid, because the uncoordinated charging coincides with the evening peak of households [4]. A controlled charging algorithm will minimize the charging impact on the grid by minimizing a parameter such as the peak load. A simple valley-filling coordinated charging algorithm at TSO-level (Transmission System Operator) is used.

For PHEVs and BEVs, this impact can be possibly further reduced by choosing an optimal battery size for each vehicle. Since every vehicle owner has a different driving behavior, it is possible to allocate a suitable battery capacity for each vehicle, which is ideal for the (daily) driven distance (see Section V). Different allocation scenarios are proposed, e.g. according to the distance to work or by imposing a proportion of electric driving (utility factor). To define a realistic driving behavior for each vehicle, an availability analysis based on stochastic data has been developed (see Section IV) [7].

However, for car manufacturers it is difficult to have an unlimited amount of battery capacities to meet the requirements of every customer. According to [3], a PHEV needs at least an AER of 10 miles $(16 \mathrm{~km})$. In this paper, PHEVs with an AER of 10 (PHEV10), 15, 20, 30, 40 and 60 miles are used. For BEVs, battery capacities with an AER of 60 (BEV60), 100, 150, 200, 250 and 300 miles are used. An analysis on the utility factor (UF) will be performed (see Section VI).

The connection of EVs to the grid enables the support of the electricity grid (V2G) by injecting power into the grid or by curtailing the charging power of the vehicles. From the 
availability analysis, it is known when a vehicle is at home. Furthermore, the state of charge (SoC) of the battery of each vehicle is known at each moment. One of the possibilities is to use PHEVs as a buffer for regulation to keep the frequency and voltage steady (matching consumption and generation). These services are only needed for a short time, but might be called upon several times a day [8]. In Section VII, the V2G availability of the fleet of EVs will be discussed.

\section{BASIC ASSUMPTIONS}

The useful battery capacity will depend on the so-called electric efficiency (in $k m / k W h$ ). The DC electric efficiency is considered in this paper. This efficiency takes the losses during the battery discharge while driving into account. A conservative electric efficiency of $5 \mathrm{~km} / \mathrm{kWh}(200 \mathrm{Wh} / \mathrm{km})$ is used. EPRI gives a value of $5.5 \mathrm{~km} / \mathrm{kWh}$ [9], while Argonne National Lab gives a value of $5.7 \mathrm{~km} / \mathrm{kWh} \mathrm{[10].}$ The charging efficiency is $90 \%$ [5], which results in an energy consumption of $222 \mathrm{Wh}$ for each electric kilometer driven.

A maximum charging power of $4 \mathrm{~kW}$ is used. This follows from the current limitations $(230 \mathrm{~V}, 20 \mathrm{~A})$ of a standard single phase outlet in buildings [11]. Three phase connections have not been taken into account. It is assumed that at home there is always a possibility to charge the batteries. Charging at work and public charging stations are not taken into account in this analysis. For re-injecting power in the distribution grid, the same power limit of $4 k W$ is used. The discharge efficiency is set at $93 \%$ [5], so $1 \mathrm{kWh}$ of stored energy in the battery will result in $0.93 \mathrm{kWh}$ of energy delivered to the grid.

\section{AVAILABILITY ANALYSIS}

An availability analysis has been developed to determine the driving behavior for each vehicle in the fleet [7]. With this analysis, it is known when each vehicle is driving, when it is at home, at work or at another activity. This analysis can be used to allocate a suitable battery capacity to each vehicle. In previous work [4] and [11], a basic availability analysis based on data from the Netherlands was used to analyze the impact of charging and discharging (V2G) of PHEV-batteries on a distribution grid. This availability analysis has been further improved and adapted with recent Flemish data on travel behavior [7]. The model is based on the statistical data from the 3rd Flemish Mobility Study (OVG3) [12]. This study, commissioned by the Flemish government, has been conducted between September 2007 and September 2008 among 8,800 people ( $\geq 6$ years old). The people surveyed, were asked to keep track of all their trips (all transport means): number of trips ${ }^{1}$ per day, the distance (duration), the motive (work, business, shopping, etc.), the departure and return times, etc. Since the travel behavior is different, a distinction has been made between weekdays and weekend days.

Since September 2008, the 4th OVG is being conducted. The results of the first two years are available in OVG4.1 and OVG4.2, but some numbers indicate that these studies might be affected by the global economic crisis and rising fuel prices,

${ }^{1} \mathrm{~A}$ trip is defined as the trip from home to the activity and back home.
TABLE I

SUBDIVISION OF GASOLINE AND DIESEL CARS ACCORDING TO THE ENGINE DISPLACEMENT [7]

\begin{tabular}{|l|c|c||c|c|}
\hline \multirow{2}{*}{$\begin{array}{l}\text { Engine } \\
\text { displacement }\end{array}$} & \multicolumn{2}{|c||}{ Distribution } & \multicolumn{2}{c|}{ Distance } \\
\cline { 2 - 5 } & Gasoline & Diesel & Gasoline & Diesel \\
\hline \hline$<\mathbf{1 . 4}$ liter & $22.64 \%$ & $4.86 \%$ & $7,960 \mathrm{~km}$ & $18,045 \mathrm{~km}$ \\
\hline $\mathbf{1 . 4}-\mathbf{2 . 0}$ liter & $14.36 \%$ & $44.40 \%$ & $9,095 \mathrm{~km}$ & $19,565 \mathrm{~km}$ \\
\hline$>\mathbf{2 . 0}$ liter & $3.00 \%$ & $10.74 \%$ & $10,335 \mathrm{~km}$ & $18,955 \mathrm{~km}$ \\
\hline \hline Total & $40 \%$ & $60 \%$ & $8,545 \mathrm{~km}$ & $19,340 \mathrm{~km}$ \\
\hline
\end{tabular}

causing a negative trend in number of trips per day and average kilometers driven per day [12].

To make the driving patterns even more realistic, the vehicles in the fleet need to be divided in more segments regarding the present vehicle characteristics. The vehicles are subdivided according to the fuel ${ }^{2}$ and engine displacement, since there is a distinction in the yearly driven kilometers (see Table I) [7]. This segmentation is at this moment only used to have more realistic driving patterns. It is supposed that this distribution of yearly driven kilometers remains, no matter which fuel will be used by PHEVs. Future research topics may include allocating different electric efficiencies to each segment.

\section{Allocation OF BATTERY CAPACITIES}

For each PHEV in the fleet, the driving patterns have been determined with the availability analysis in Section IV. With these results, battery sizes will be allocated to the fleet in this section. Different allocation scenarios are proposed.

\section{A. Scenarios for the allocation of battery sizes}

In this paper, four different allocation scenarios for the batteries are proposed:

- Scenario 1: Allocation of the battery capacity according to the average of the longest trip each day in one simulated year.

- Scenario 2: Allocation of the battery capacity according to the distance to and from work (and scenario 1 for vehicle owners who do not work).

- Scenario 3: Allocation of the battery capacity according to the distance to work (and scenario 1 for vehicle owners who do not work).

- Scenario 4: Allocation of the battery capacity according to the required utility factor (proportion electric driving) that must be met during the simulated year.

The four scenarios will be tested separately and the results will be used to compare the different scenarios on the utility factor (UF), the extra power consumption and the V2G availability.

The first three scenarios are based on a certain daily distance (criterion distance) traveled by each vehicle. Since these scenarios do not guarantee sufficient battery capacity for BEVs to drive purely electrically, these scenarios can only be applied to PHEVs. Vehicle owners who only drive a limited number of kilometers, namely a criterion distance of less than $4 \mathrm{~km}$ (2.5 miles) are divided into a HEV-category (AER of

\footnotetext{
${ }^{2}$ Liquefied petroleum gas (LPG) has been neglected.
} 
TABLE II

RESULTS OF THE BATTERY ALLOCATION FOR PHEVS FOR THE DIFFERENT SCENARIOS, INCLUDING THE AVERAGE USEFUL BATTERY CAPACITY

\begin{tabular}{|l|c||c|c|c|c|c|c|c||c|}
\hline & $\begin{array}{c}\text { Required } \\
\text { utility factor }\end{array}$ & HEV & PHEV10 & PHEV15 & PHEV20 & PHEV30 & PHEV40 & PHEV60 & $\begin{array}{c}\text { Average useful } \\
\text { battery capacity }\end{array}$ \\
\hline \hline Scen. 1 & - & - & $35.6 \%$ & $2.0 \%$ & $49.2 \%$ & $12.2 \%$ & $1.0 \%$ & - & $5.72 \mathrm{kWh}$ \\
\hline Scen. 2 & - & $1.6 \%$ & $45.6 \%$ & $5.4 \%$ & $36.6 \%$ & $8.2 \%$ & $1.6 \%$ & $1.0 \%$ & $5.28 \mathrm{kWh}$ \\
\hline Scen. 3 & - & $3.8 \%$ & $51.0 \%$ & $2.8 \%$ & $34.6 \%$ & $7.8 \%$ & - & - & $4.77 \mathrm{kWh}$ \\
\hline Scen. 4 & $50 \%$ & - & $34.0 \%$ & $4.0 \%$ & $32.8 \%$ & $28.4 \%$ & $0.8 \%$ & - & $6.24 \mathrm{kWh}$ \\
\hline Scen. 4 & $60 \%$ & - & $9.2 \%$ & $27.8 \%$ & $1.0 \%$ & $59.2 \%$ & $2.2 \%$ & $0.6 \%$ & $7.82 \mathrm{kWh}$ \\
\hline Scen. 4 & $70 \%$ & - & - & $27.2 \%$ & $10.0 \%$ & $9.8 \%$ & $51.4 \%$ & $1.6 \%$ & $9.83 \mathrm{kWh}$ \\
\hline Scen. 4 & $80 \%$ & - & - & $0.8 \%$ & $26.0 \%$ & $11.2 \%$ & $10.0 \%$ & $52.0 \%$ & $14.12 \mathrm{kWh}$ \\
\hline Scen. 4 & $90 \%$ & - & - & - & - & $28.4 \%$ & $8.8 \%$ & $62.8 \%$ & $16.00 \mathrm{kWh}$ \\
\hline
\end{tabular}

1 mile). Vehicles whose criterion distance is e.g. 24 and 26 miles, will be respectively divided into PHEV20 and PHEV30.

In the 4th scenario, a required UF is given that must be met during the simulated year. When e.g. a UF of $50 \%$ is required, half of the total distance traveled during the simulated year must be driven electrically. Only UFs $\geq 50 \%$ will be discussed, whereas a required UF of $100 \%$ coincides with a BEV.

In this paper, it is only possible to charge the vehicles at home. In future work, the effect of the possibility to charge at work will be discussed as (i) a range-extender and (ii) the possibility to reduce the battery capacity to attain a same UF.

\section{B. Results on the allocation of the batteries}

Here, the results will be discussed for the battery allocation according the different scenarios for PHEVs and BEVs. For each scenario, two peaks in the allocation can be noticed. For instance in scenario 1, most vehicles are allocated in PHEV10 and PHEV20 (see Table II). This can be explained with Table I, which states that gasoline and diesel vehicle have a different behavior regarding the yearly driven distance.

1) PHEVs: The results of the battery allocation, including the average useful battery capacity of a fleet of PHEVs, for the different scenarios are given in Table II. The allocation of battery capacities in the 2nd and 3rd scenario is based on the work trip. The distance to work is generally well known in advance, which means these scenarios will be easier applicable, whereas the other scenarios need more information on the yearly driving patterns. The 3rd scenario results in the smallest average battery capacity of the investigated scenarios, but also in more and relatively deeper discharges per day, so the battery wear is much higher. However, the difference of the average battery capacity between scenario 2 and 3 is relatively small because only $38 \%^{3}$ of the vehicles is used for work trips.

Generally, almost all vehicles are allocated in a PHEV20category or lower in the first three scenarios. The results in Table II show that about $90 \%$ does not need more than $6.4 \mathrm{kWh}$ of battery capacity in these cases. In scenario 2 and 3, there are even a number of vehicle owners who are allocated as a HEV. In scenario 1, the allocation is based on the average of the longest trips. As seen in Table II, this does

\footnotetext{
${ }^{3}$ Future work will also take the large share of company cars in Flanders into account. In that case, the differences may become larger.
}

not automatically mean that more vehicles are allocated into larger categories ( $\geq$ PHEV30) compared to the 2nd scenario.

Table II also gives the results for the 4th scenario for a required UF ranging from $50 \%$ to $90 \%$. From these results it follows that every PHEV is at least a PHEV10. Since every PHEV has to meet the required yearly UF, the battery capacities will be remarkably higher than in the previous scenarios. Suppose a PHEV needs a battery capacity for 21 electric miles $( \pm 34 \mathrm{~km})$ a day to have a UF of $50 \%$, it will be allocated as a PHEV30, since a PHEV20 does not meet the requirements. Furthermore, the longer trips will play a more important role than in the first three scenarios, since the battery will have to be dimensioned for these trips, whereas in the first three scenarios only the average of the longest trip ${ }^{4}$ was of importance. This is shown in Table II when the required UF becomes large.

From these results, it follows that to obtain a UF of $90 \%$ for each vehicle, it seems possible to do this with battery sizes smaller than $19.31 \mathrm{kWh}$ (AER $\cong 60$ miles), i.e. the introduction of a PHEV with a larger AER is not necessary. As mentioned before, a battery cannot be fully depleted to prevent deep discharge cycles with a high battery wear. If e.g. there is an unused battery capacity of $30 \%$, this corresponds with an average total battery capacity of $\frac{19.31 \mathrm{kWh}}{0.7}=27.59 \mathrm{kWh}$. However, the results on the utility factor (see Section VI) show that for some vehicles a PHEV60 may be too small to obtain a UF of $90 \%$. However, it is clear that high UFs can, in many cases, be obtained with rather limited battery capacities.

2) BEVs: BEVs only have an electric motor for propulsion, which means these vehicles require a utility factor of $100 \%$. Their batteries are dimensioned for that one day with the largest total distance traveled. Fig. 1 (a) shows the distribution of these distances for the fleet for which the batteries have to be dimensioned. Results show that a PHEV60, as used for PHEVs, is not at all enough for BEVs. The minimum battery capacity is designed for the vehicle which has a maximum daily distance of about $80 \mathrm{~km}(16 \mathrm{kWh})$, whereas the maximum battery capacity is designed for a maximum distance of almost $460 \mathrm{~km}(92 \mathrm{kWh})$. Fig. 1 (b) shows the allocation of the fleet in different BEV-categories. About $80 \%$ of the vehicles is categorized in BEV100 to BEV200. The average battery capacity of the fleet is $53.70 \mathrm{kWh}$, which is

\footnotetext{
${ }^{4}$ For vehicles not used for work in scenario 2 and 3 .
} 
TABLE III

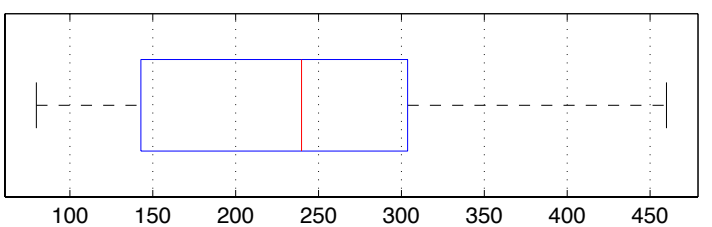

(a) Distribution of longest distance (on one day) for each vehicle [km]

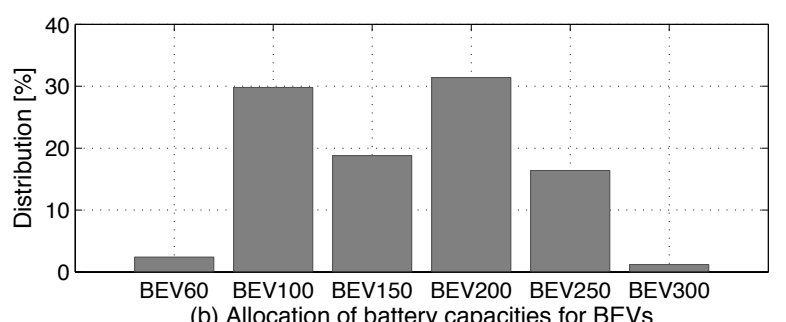

Fig. 1. Results of the battery allocation for BEVs

remarkably higher than in the case of the PHEV-allocation.

\section{ANALYsis OF THE Utility FACtOR AND EXTRA POWER CONSUMPTION}

The battery allocation scenarios for PHEVs and BEVs are discussed in this section on the UF and the extra household power consumption. The batteries are fully ${ }^{5}$ charged at home around the moment with the smallest power demand in the grid (including the load of the other EVs). Mainly, the charging takes places overnight, but it allows vehicles to charge during the day when they are used for trips during the night.

\section{A. Utility Factor}

EPRI has defined the utility factor (UF) for individual PHEVs [13]. They define the UF as the ratio of the annual electric kilometers over the annual vehicle kilometers traveled.

Table III shows the results on the average UF for the fleet for each scenario. The average UF is higher with higher average battery capacities. In the three first scenarios, the UF varies from $43 \%$ (scenario 3) to $53.4 \%$ (scenario 1). Note that the difference between the 2nd and 3rd scenario is rather limited, since only a limited number of vehicles is used for work.

In scenario 4 , the battery allocation follows from the required UF. Since every PHEV has to meet the required yearly UF, these PHEVs will be over dimensioned because of the limited number of battery sizes. For this reason, the actual UF will be higher than the required one. The only exception is for a UF of $90 \%$, then the UF is just under the required one. This can be explained by the fact that PHEV60 is not enough for some vehicles to obtain a UF of $90 \%$.

\section{B. Extra household power consumption}

The yearly average power consumption of a Flemish household is $3,500 \mathrm{kWh}$ [6]. Suppose only one fixed battery capacity of $8.8 \mathrm{kWh}$ (example from [4]) is available for PHEVs, this

\footnotetext{
${ }^{5}$ As far as the vehicle is at home for a sufficient time.
}

AVERAGE UTILITY FACTOR, EXTRA AVERAGE YEARLY POWER CONSUMPTION OF EVERY VEHICLE IN THE FLEET AND THE POWER CONSUMPTION PER USEFUL KWH BATTERY CAPACITY

\begin{tabular}{|l|c||c|c|c|}
\hline & $\begin{array}{c}\text { Required } \\
\text { UF }\end{array}$ & $\begin{array}{c}\text { Average } \\
\text { UF }\end{array}$ & $\begin{array}{c}\text { Yearly power } \\
\text { consumption (1) }\end{array}$ & $\frac{(\mathbf{1})}{k W h_{\text {battery }}}$ \\
\hline \hline \multicolumn{5}{|c|}{ PHEV } \\
\hline Scen. 1 & - & $53.4 \%$ & $1,782 k W h$ & 311.5 \\
\hline Scen. 2 & - & $47.6 \%$ & $1,658 k W h$ & 314.0 \\
\hline Scen. 3 & - & $43.0 \%$ & $1,507 k W h$ & 315.9 \\
\hline Scen. 4 & $50 \%$ & $56.1 \%$ & $1,891 k W h$ & 303.0 \\
\hline Scen. 4 & $60 \%$ & $65.8 \%$ & $2,202 k W h$ & 281.6 \\
\hline Scen. 4 & $70 \%$ & $74.2 \%$ & $2,497 k W h$ & 254.0 \\
\hline Scen. 4 & $80 \%$ & $85.3 \%$ & $2,876 k W h$ & 203.7 \\
\hline Scen. 4 & $90 \%$ & $89.7 \%$ & $2,978 k W h$ & 186.1 \\
\hline \hline \multicolumn{5}{|c|}{ BEV } \\
\hline Scen. 4 & $100 \%$ & $100 \%$ & $3,348 k W h$ & 62.3 \\
\hline
\end{tabular}

would double the yearly power consumption (with a charging efficiency of 90\% [5]) in case the battery is fully charged every day. When taking into account the driving behavior of the fleet, the extra household power consumption is limited to $2,222 \mathrm{kWh}$, since the batteries are not fully discharged every day. In this section the impact of the four scenarios of PHEVs and BEVs on the household power consumption is discussed.

1) PHEVs: The first three scenarios are based on a certain daily distance driven by each PHEV. The average battery capacity is relatively limited: from $4.77 \mathrm{kWh}$ (3rd scenario) to $5.72 \mathrm{kWh}$ (1st scenario). The extra power consumption is on average 1, $507 \mathrm{kWh}$ to $1,782 \mathrm{kWh}$ per vehicle (see Table III). In scenario 4, the extra household power consumption is limited from 1,891 $\mathrm{kWh}$ (required UF of 50\%) to 2, $978 \mathrm{kWh}$ (required UF of 90\%) per vehicle. These results show that the larger the average battery capacity, the less optimal the battery is used. This is also shown by the ratio of the yearly power consumption and the average useful battery capacity in Table III. For instance, for a required UF of $50 \%$ and $90 \%$, there is a yearly consumption of respectively $303 \mathrm{kWh}$ and $186 k W h$ for each installed useful $k W h$ of battery capacity.

2) BEVs: The average battery capacity for a fleet of BEVs is quite high $(53.70 \mathrm{kWh})$. This means the maximum distance during one day for each vehicle is high. However, the average daily driven distance is much lower. On average, the yearly power consumption is $3,348 \mathrm{kWh}$ for each $\mathrm{BEV}$, which is slightly higher than for PHEVs with a required UF of $90 \%$. Each vehicle drives on average 15,000 km per year, which corresponds with this extra power consumption $\left(\frac{15000 \mathrm{~km}}{5 \mathrm{~km} / \mathrm{kWh} \cdot 0.90}=\right.$ $3,333 k W h$ ). The yearly power consumption per installed useful $k W h$ of battery capacity is only $62 k W h$.

\section{ANALYSIS OF THE V2G-FLEXIBILITY}

The connection of EVs to the grid enable the support of the electricity grid by re-injecting power into the grid or lowering the charging power of the vehicles (V2G). EVs can be used to match supply, both central and distributed generation, and demand of electricity. Since EVs are able to act quickly, one of the possibilities is to use EVs as a buffer for regulation 


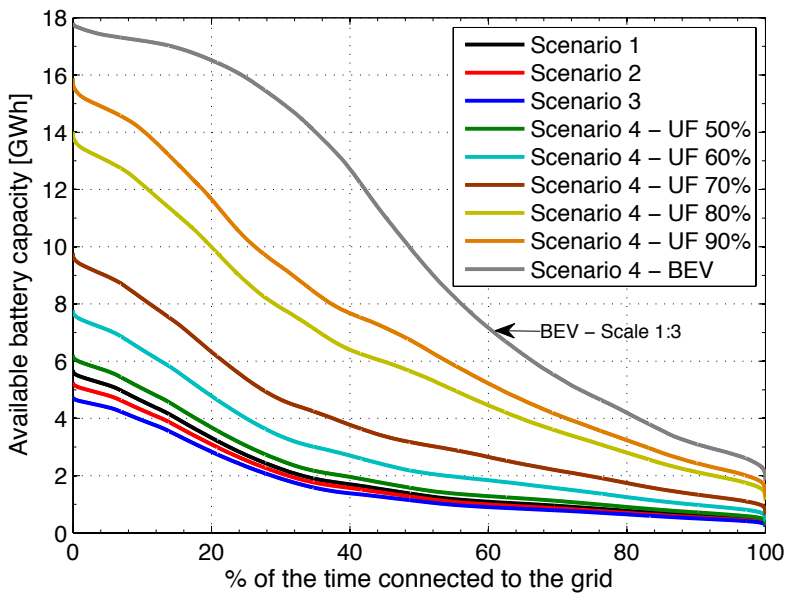

Fig. 2. Available battery capacity for a fleet of 1,000,000 EVs for the different scenarios for PHEVs (scale 1:1) and BEVs (scale 1:3) during one year

to keep the frequency and voltage steady in real-time. These services are needed for a short time (one up to a few minutes), but might be called upon several tens to hundreds times a day. Regulation can be split into up regulation (less charging or injecting power in the grid) or down regulation (more charging or injecting less power in the grid) [8]. Only up regulation will be considered here by taking a look at the available battery capacity, the total charge power and total available discharge power of a fleet of PHEVs or BEVs during a year.

Transport \& Mobility used the TREMOVE model to predict the future Belgian vehicle fleet [14]. By 2020, they predict a share of $18 \%$ HEVs (about 1,000,000 HEVs) in the Belgian fleet. Here, the analysis of the V2G-flexibility will be performed with a fleet of 1 million vehicles. It is assumed that the predicted fleet of HEVs are all PHEVs, respectively BEVs.

\section{A. Available battery capacity}

Fig. 2 shows for how much time in one year a certain total battery capacity of the fleet of PHEVs, respectively BEVs is available, when connected to the grid at home. For all scenarios, the minimum available battery capacity varies from $4.5 \%(0.26 G W h)$ in scenario 1 to $9.6 \%(5.16 G W h)$ in scenario 4 (BEVs) of the total fleet battery capacity. This means there is not a single moment the fleet has no possibilities to support the grid by re-injecting power when connected to the grid. Secondly, to obtain high UFs, the vehicle fleet requires a higher average battery capacity. This can be seen as a major disadvantage because of the high cost and weight of batteries. But Fig. 2 shows that a large energy reserve is available to re-inject into the grid.

\section{B. Up regulation: Charging power}

Fig. 3 shows for each scenario for PHEVs and BEVs for how many time in the simulated year a certain charging power is reached. This can also be interpreted as the possible up regulation by lowering the EV charging power. The results are highly dependent on the scenario. There is also a difference between the PHEV-scenarios and the BEV-scenario.

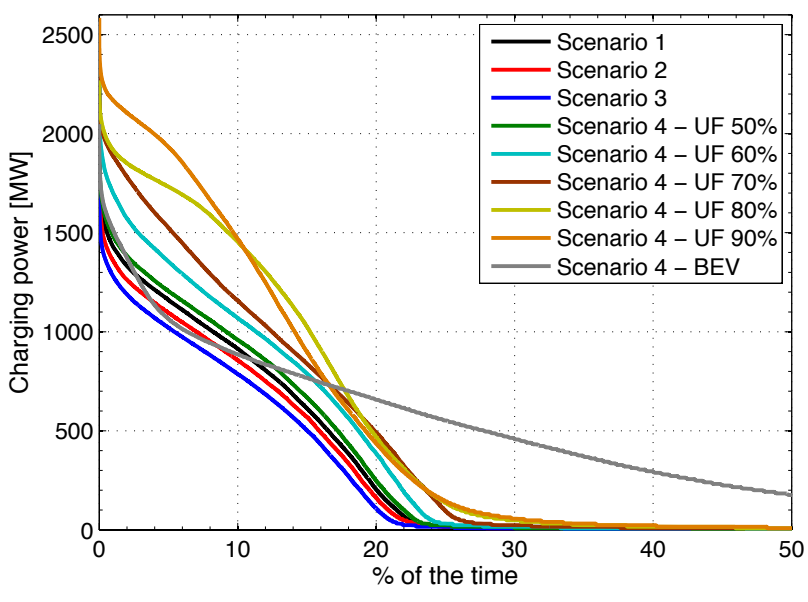

Fig. 3. Charging load diagram for a fleet of 1,000,000 EVs for the different scenarios for PHEVs and BEVs during one year

1) PHEVs: It is clear from this figure, that only during a limited time of the year PHEVs are charged, namely a maximum of about $20 \%$ (scenario 3) to about $30 \%$ (scenario 4 with required UF of $90 \%$ ). It is obvious that a larger battery capacity needs a longer time to charge. Secondly, the higher the average battery capacity for PHEVs, the higher the simultaneous charging power of the total fleet will be. This can be explained by the fact that the charging time for each vehicle is higher, resulting in more simultaneous charging.

2) $B E V s$ : For the case of a fleet of $1,000,000 \mathrm{BEVs}$, the distribution of the fleet charging power is more spread out compared to the PHEV-scenarios. At each moment in the simulated year, one or more vehicles are charging. Since the batteries of these vehicles are much larger than in the PHEVscenarios, the charging time can become very large on those days the batteries are used more, resulting in this spread.

\section{Up regulation: Injecting power}

The available discharge power of each EV depends on the energy in its battery. Here, the available discharge power of the fleet in time will be discussed. To use PHEVs or BEVs as a buffer for regulation, it is important to have an idea on the available fleet power, since for regulation, it is necessary to deliver enough power for a short time (one up to a few minutes), rather than delivering energy for a longer time.

The results, given in Fig. 4, show that the instantaneous available fleet power, when connected to the grid at home, is almost independent of the average installed battery capacity of the fleet. The scenarios with a larger average battery capacity, have a slightly higher fleet power availability. This independence can be explained by the fact that the discharging power is limited to $4 \mathrm{~kW}$. To re-inject $4 k W$ in the grid for one minute, only $0.072 k W h\left(=\frac{4 k W}{60 \times 0.93}\right)$ is needed. The figure only shows the available fleet power for the moments the vehicles are connected to the grid at home.

In none of the investigated scenarios, there is a single moment with no available discharging power in the fleet. The minimum available fleet power is about $11 \%$ (on average 


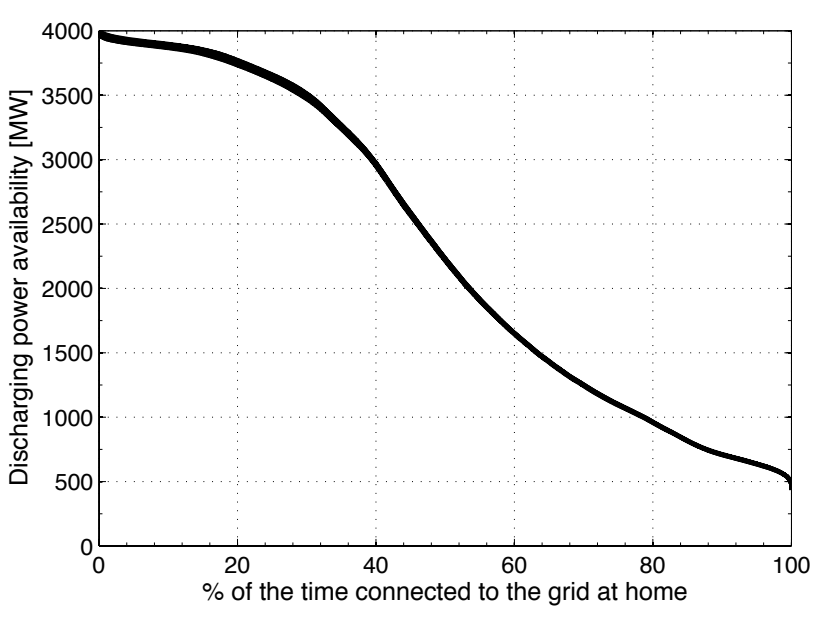

Fig. 4. Instantaneous discharging power availability for a fleet of $1,000,000$ EVs when connected to the grid during one year

$0.44 k W$ per EV). There are even some moments in the simulated year, the maximum fleet power is available.

\section{CONCLUSIONS}

Based on Flemish statistical data on transport behavior, a fleet of electric vehicles with realistic driving behavior is created. This is used for the optimal battery sizing of PHEVs and BEVs. Several battery allocation scenarios are proposed based on a certain trip distance or a required utility factor (UF). To obtain a high UF for PHEVs, the average useful battery capacity of the fleet remains relatively small (e.g. $16 \mathrm{kWh}$ for a UF of 90\%). For BEVs, the average useful battery is remarkably higher $(53.7 \mathrm{kWh})$ since the batteries have to be dimensioned for the day with the most kilometers traveled.

Since batteries, especially for scenarios with larger battery capacities, are not fully discharged every day, the average yearly power consumption for EV-charging is limited to 3, $348 \mathrm{kWh}$ (BEVs). In case of PHEVs, during 20\% to $30 \%$ of the time, charging power can be lowered as a V2G-service. When connected to the grid, there is not a single moment there is no available battery capacity in the fleet to support the grid. Results also show that the discharging power availability is almost independent of the installed battery capacity.

\section{REFERENCES}

[1] World Energy Outlook. International Energy Agency.

[2] B. K. Sovacool and R. F. Hirsh, "Beyond batteries: An examination of the benefits and barriers to plug-in hybrid electric vehicles (PHEVs) and a vehicle-to-grid (V2G) transition," Energy Policy, vol. 37, no. 3, pp. 1095-1103, March 2009.

[3] S. G. Wirasingha, N. Schofield, and A. Emadi, "Plug-In Hybrid Electric Vehicle Developments in the US: Trends, Barriers and Economic Feasibility," in IEEE Vehicle Power and Propulsion Conference, Harbin, China, September 2008, pp. 1-8.

[4] K. Clement-Nyns, E. Haesen, and J. Driesen, "The Impact of Charging Plug-in Hybrid Electric Vehicles on a Residential Distribution Grid," IEEE Transactions on Power Systems, vol. 25, no. 1, pp. 371-380, February 2010.

[5] F. Geth, J. Tant, D. Six, P. Tant, T. De Rybel, and J. Driesen, "Technoeconomical and life expectancy modeling of battery energy storage systems," in $21^{\text {st }}$ International Conference and Exhibition on Electricity Distribution - CIRED, Frankfurt, Germany, June 6-9 2011.
[6] (Last accessed: 2011, Jul. 28) Flemish Regulator for the Energy and Gas markets (VREG). [Online]. Available: http://www.vreg.be

[7] J. Van Roy, N. Leemput, S. De Breucker, P. Tant, and J. Driesen, "Study on the Availability Analysis and Energy Consumption of Electric Vehicles," in European Electric Vehicle Congress, Brussels, Belgium, October 26-28, 2011, Accepted for publication.

[8] W. Kempton and J. Tomic, "Vehicle-to-grid power fundamentals: Calculating capacity and net revenue," Journal of Power Sources, vol. 144 no. 1, pp. 268-279, June 2005.

[9] M. Duvall, "Comparing the Benefits and Impacts of Hybrid Electric Vehicle Options for Compact Sedan and Sport Utility Vehicles," Electric Power Research Institute, Tech. Rep., 2002.

[10] A. Rousseau, N. Shidore, R. Carlson, and P. Nelson, "Research on PHEV Battery Requirements and Evaluation of Early Prototypes," in $7^{\text {th }}$ Advanced Automotive Battery Conference. Long Beach, California: Argonne National Laboratory, May 16-18 2007.

[11] K. Clement-Nyns, E. Haesen, and J. Driesen, "The Impact of Vehicleto-Grid on the Distribution Grid," Electric Power Systems Research Journal, vol. 81, no. 1, pp. 185-192, January 2011.

[12] Mobiliteit en Openbare Werken Vlaanderen - Mobiel Vlaanderen. (Last accessed: 2011, Jul. 28) Onderzoek Verplaatsingsgedrag Vlaanderen. [Online]. Available: http://www.mobielvlaanderen.be/ovg/

[13] M. Duvall and E. Knipping, "Environmental Assessment of Plug-In Hybrid Electric Vehicles," Electric Power Research Institute, Tech. Rep., July 2007.

[14] S. Logghe, B. Van Herbruggen, and B. Van Zeebroeck, "Emissions of road traffic in Belgium," Transport \& Mobility Leuven, Tech. Rep., January 2006

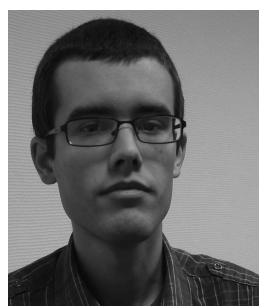

Juan Van Roy (S'09) was born in Belgium in 1987. He received the M.Sc. degree in Electrical Engineering, with specialization in energy, from the Katholieke Universiteit Leuven (K.U.Leuven), Belgium, in 2010, where he is currently working towards the Ph.D. degree.

Currently, he is working as a research assistant with the division ESAT-ELECTA (K.U.Leuven). His research interests include controlling the impact of charging (hybrid) electric vehicles on the grid and the electrical aspect of energy intelligent buildings.

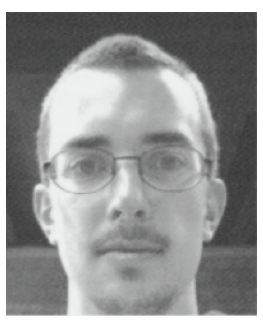

Sven De Breucker was born in 1980 in Belgium. $\mathrm{He}$ received the M.Eng. degree in Electromechanics from the De Nayer University College, Belgium, in 2002, and the M.Sc. degree in Electrotechnical Engineering from the Katholieke Universiteit Leuven (K.U.Leuven), Leuven, Belgium, in 2007.

Currently, he is working towards the Ph.D. degree with VITO and the division ESAT-ELECTA (K.U.Leuven) on DC/DC convertors and batteries. His research interests include power electronics and electric drives.

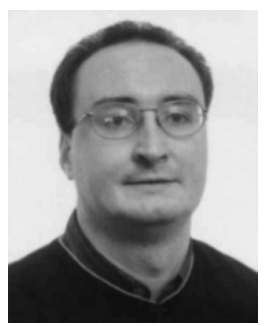

Johan Driesen (S'93 - M'97) was born in 1973 in Belgium. He received the M.Sc. and Ph.D. degrees in Electrical Engineering from the Katholieke Universiteit Leuven (K.U.Leuven), Leuven, Belgium, in 1996 and 2000, respectively.

Currently, he is a Professor with the K.U.Leuven and teaches power electronics and electric drives. In 2000, he was with the Imperial College of Science, Technology and Medicine, London, U.K. In 2002, he was with the University of California, Berkeley.

Currently, he conducts research on distributed generation, power electronics, and its applications. 